\title{
Comparação entre os dispositivos intrauterinos de cobre e hormonal: uma revisão narrativa
}

\author{
Comparison between copper and hormonal intrauterine devices: a narrative review \\ Comparación entre dispositivos intrauterinos hormonales y de cobre: una revisión \\ narrativa
}

Nathalia Coelho Slywitch ${ }^{1 *}$, Bruna Pereira Alves ${ }^{1}$, Evelli Aline de Paula Martins ${ }^{1}$, Júlia Veloso Romão ${ }^{1}$, Marina Silveira Amorim ${ }^{1}$, Mariana Por-Deus Vilela ${ }^{1}$, Morgana Soares Borges ${ }^{1}$, Nathália Laport Guimarães Borges ${ }^{1}$, Valério Ferreira da Cunha Netoํㄹ , Danielle Fiorin Ferrari Novais².

\section{RESUMO}

Objetivo: Comparar e revisar as diversas características do Dispositivo Intrauterino (DIU) de cobre com o DIU hormonal, apontando seus mecanismos de ação, eficácias, contraindicações, vantagens e desvantagens, além de sua relação com o HIV. Revisão Bibliográfica: O DIU é um método anticoncepcional altamente eficaz, com taxas de falha em torno de 1\%. Não é abortivo e não interrompe a gravidez. O principal mecanismo de ação do DIU de cobre é prejudicar a fecundação do oócito pelo espermatozóide. O DIU hormonal, por sua vez, constituído de levonorgestrel, tem como principal mecanismo de ação o espessamento do muco cervical (impedindo a passagem do esperma pelo colo do útero) e a diminuição da motilidade dos espermatozóides. A taxa de eficácia do DIU hormonal é muito alta, podendo ser representada através do índice de Pearl 0,1. Já o DIU de cobre, que é provavelmente o mais utilizado em todo mundo, tem durabilidade de dez anos e o índice de Pearl muito baixo, ocorrendo uma gravidez ou menos em cada 100 mulheres durante o primeiro ano de uso. Considerações finais: Os dispositivos intrauterinos hormonais e de cobre apresentam características específicas em relação à sua indicação, eficácia, repercussões sistêmicas e principalmente os efeitos colaterais.

Palavras-chave: Dispositivos intrauterinos, Contracepção reversível de longo prazo, Dispositivos intrauterinos de cobre.

\begin{abstract}
Objective: Compare and review the various characteristics of the copper Intra uterine device (IUD) with the hormonal IUD, pointing out its mechanisms of action, efficacy, contraindications, advantages and disadvantages, in addition to their relationship with HIV. Bibliographic Review: The IUD is a highly effective contraceptive method, with failure rates of around $1 \%$. It is not abortive and does not terminate the pregnancy. The main mechanism of action of the copper IUD is to impair the fertilization of the oocyte by the sperm. The hormonal IUD, in turn, made up of levonorgestrel, has as its main mechanism of action the thickening of the cervical mucus (preventing the passage of cervical mucus). sperm through the cervix) and decreased sperm motility. The rate of effectiveness of the hormonal IUD is very high and can be represented by the Pearl index 0.1 . The copper IUD, which is probably the most used worldwide, has a ten-year durability and the Pearl index is very low, with one pregnancy or less in every 100 women during the first year of use. Final considerations: The hormonal and copper intrauterine devices have specific characteristics in relation to their indication, efficacy, systemic repercussions and mainly the side effects.
\end{abstract}

Key words: Intrauterine devices, Long-acting reversible contraception, Intrauterine devices copper.

${ }^{1}$ Universidade IMEPAC (IMEPAC), Araguari - MG. *E-mail: nathaliaslywitch@hotmail.com

${ }^{2}$ Faculdade de Medicina Multivix, Vitória - ES.

SUBMETIDO EM: 4/2021

ACEITO EM: 5/2021

PUBLICADO EM: 5/2021 


\section{RESUMEN}

Objetivo: Comparar y revisar las diversas características del Dispositivo Intrauterino (DIU) de cobre con el DIU hormonal, señalando sus mecanismos de acción, eficacia, contraindicaciones, ventajas y desventajas, además de su relación con el VIH. Revisión bibliográfica: EI DIU es un método anticonceptivo muy eficaz, con tasas de fracaso de alrededor del 1\%. No es abortivo y no interrumpe el embarazo. El principal mecanismo de acción del DIU de cobre es perjudicar la fertilización del ovocito por parte de los espermatozoides. EI DIU hormonal, a su vez, compuesto por levonorgestrel, tiene como principal mecanismo de acción el espesamiento del moco cervical (impidiendo el paso de moco cervical), esperma a través del cuello uterino) y disminución de la motilidad de los espermatozoides. La tasa de efectividad del DIU hormonal es muy alta y puede representarse mediante el índice de Pearl 0.1. EI DIU de cobre, que probablemente sea el más utilizado en todo el mundo, tiene una durabilidad de diez años y el índice de Pearl es muy bajo, con un embarazo o menos de cada 100 mujeres durante el primer año de uso. Consideraciones finales: Los dispositivos intrauterinos hormonales y de cobre tienen características específicas en relación a su indicación, eficacia, repercusiones sistémicas y principalmente los efectos secundarios.

Palabras clave: Dispositivos intrauterinos, Anticoncepción reversible de larga duración, Dispositivos intrauterinos de cobre.

\section{INTRODUÇÃO}

Os contraceptivos reversíveis de ação prolongada, também conhecidos como Long-Acting Reversible Contraception (LARC), referem-se a métodos que são eficazes por longo período de tempo e não necessitam de ação do usuário. Incluem, além do implante subdérmico, os Dispositivos Intrauterinos (DIU) (ADEYEMIFOWODE OA e BERCAW-PRATT JL, 2019).

Além de serem apontados como um dos métodos mais eficazes de contracepção, os DIUs têm se mostrado econômicos quando comparados com pílulas anticoncepcionais orais, adesivos, anéis e injeções (SANDERS JN, et al., 2018).

Os mecanismos de ação do DIU diferem conforme sua composição (cobre ou levonorgestrel), mas de uma maneira geral, se resumem em retardar ou acelerar o transporte do embrião inicial por meio das tubas uterinas, danificar ou destruir o embrião inicial antes que ele alcance o útero, além de prevenir sua implantação (STANFORD JB e MIKOLAJCZYK RT, 2002; ADEYEMI-FOWODE OA e BERCAW-PRATT JL, 2019).

Ainda que seja um método contraceptivo de uso generalizado em todo o mundo, o uso do DIU é menos difundido em países desenvolvidos (9\%) do que naqueles em desenvolvimento (14\%), onde os métodos mais comumente usados são as pílulas anticoncepcionais orais e os preservativos. Dentre os países em desenvolvimento, seu uso ainda varia, sendo mais baixo nos menos desenvolvidos devido a falta de disponibilidade tanto de material, quanto de profissionais de saúde habilitados para a sua inserção (ARROWSMITH ME, et al., 2012).

O DIU é um método anticoncepcional altamente eficaz, com taxas de falha em torno de $1 \%$. Não é abortivo e não interrompe a gravidez. Pode estar associado a inúmeros efeitos adversos, como alterações no padrão de sangramento menstrual (que acaba aumentando), dor pélvica, expulsão e efeitos relacionados ao hormônio da progesterona, que incluem acne, dores de cabeça, náuseas, sensibilidade mamária e alterações de humor (ADEYEMI-FOWODE OA e BERCAW-PRATT JL, 2019).

O objetivo do presente estudo é, a partir de uma revisão narrativa, comparar as diversas características do DIU de cobre com o DIU hormonal, apontando seus mecanismos de ação, eficácias, contraindicações, vantagens e desvantagens, além de sua relação com o HIV. 


\section{REVISÃO BIBLIOGRÁFICA}

\section{Mecanismo de ação DIU de cobre x DIU hormonal}

O principal mecanismo de ação do DIU de cobre é dificultar a fecundação do oócito pelo espermatozoide. Os íons de cobre são tóxicos para os espermatozoides e oócitos e ficam acumulados no trato reprodutivo, incluindo as tubas uterinas. Não interfere, portanto, no desenvolvimento folicular e/ou ovulação (DINEHART E, et al., 2020).

Estudo in vitro sugere que o efeito do cobre nas células estromais do endométrio humano decidualizadas interrompe a assinatura gênica (49 dos 192 genes) ligada à receptividade do endométrio. 19 dos 49 genes desregulados ligados à receptividade endometrial estão implicados na fisiopatologia da endometriose (DINEHART E, et al., 2020).

Os autores cogitam que a alteração na assinatura gênica pelo cobre, explica um dos efeitos colaterais do DIU de cobre que é o aumento do sangramento menstrual, através da alteração da vascularização sub endometrial. Faz-se necessário estudos in vivo para confirmar este efeito do DIU de cobre no endométrio (DINEHART E, et al., 2020).

Um estudo de coorte prospectivo com uma amostra de 281 adolescentes em comparação com 571 mulheres entre 20 a 30 anos, concluiu que as usuárias de DIU de cobre apresentaram maiores taxas de dor, sangramento, expulsão e deslocamento do dispositivo do que as usuárias de DIU hormonal (ADEYEMIFOWODE OA e BERCAW-PRATT JL, 2019).

Entre as vantagens conhecidas do DIU de cobre, incluem-se o uso de um método contraceptivo não hormonal e a sua capacidade em fornecer anticoncepção de emergência por até cinco dias após relação sexual desprotegida, além de ser considerado altamente eficaz na prevenção de gravidez, com taxa de falha de apenas 1\% (ADEYEMI-FOWODE OA e BERCAW-PRATT JL, 2019; HSIA JK e CREININ MD, 2016; ARROWSMITH ME, et al., 2012). Em estudos realizados por Bahamondes L, et al. (2015), observaram uma ligação entre um efeito protetor para o câncer endometrial e o uso de DIU de cobre. O mecanismo de ação ainda não foi totalmente descrito. Sugere-se que o DIU de cobre induz uma alteração de resposta do endométrio aos esteróides ovarianos e provoca uma inflamação crônica endometrial, que reduz a possibilidade de câncer.

O DIU hormonal, por sua vez, constituído de levonorgestrel, tem como principal mecanismo de ação o espessamento do muco cervical (impedindo a passagem do esperma pelo colo do útero) e a diminuição da motilidade dos espermatozoides (DINEHART E, et al., 2020). Existem atualmente 4 dispositivos hormonais disponíveis e aprovados pela Food and Drug Administration (FDA) dos EUA, que são: Mirena, Skyla, Liletta e Kyleena. Todos em forma de T, com uma manga de polidimetilsiloxano, que contém levonorgestrel na haste (ADEYEMI-FOWODE OA e BERCAW-PRATT JL, 2019). Possui um reservatório com $52 \mathrm{mg}$ de levonorgestrel, mede $32 \mathrm{~mm}$ de comprimento e libera $20 \mu \mathrm{g}$ de levonorgestrel por dia (FEBRASGO, 2018).

O DIU de levonorgestrel mostrou-se eficaz no tratamento de dismenorreia. Um estudo realizado com 200 mulheres de 18 a 25 anos, usuárias de pílulas anticoncepcionais orais combinadas ou DIU hormonal, sugeriu que o DIU se mostrou mais eficaz no tratamento da dismenorreia, com uma diminuição de 18,3\% (ADEYEMIFOWODE OA e BERCAW-PRATT JL, 2019). Além de atuarem para redução de dismenorreia, aumentam os níveis de hemoglobina em mulheres com adenomiose. Ademais, representam uma alternativa para pacientes com anemia devido a sangramento uterino anormal e que devem realizar histerectomia, porém não são candidatas cirúrgicas ideais (HSIA JK e CREININ MD, 2016).

\section{Indicações}

O DIU é indicado para todas as mulheres que desejam contracepção eficaz, incluindo adolescentes, no pós-aborto e pós-parto e em mulheres que possuem comorbidades e contraindicação aos métodos contendo estrogênio. Dessa forma, a grande abrangência do dispositivo está relacionada com o pequeno número de contraindicações que o método possui. Durante alguns anos, mulheres que nunca tiveram filhos tinham contraindicação ao método. Porém, estudos recentes vêm elucidando que não há dificuldade na inserção dos 
DIUs em mulheres nuligestas, mas neste grupo há um aumento nas taxas de expulsão durante os seis primeiros meses após a inserção do dispositivo (FEBRASGO, 2016).

O DIU de cobre é uma opção para mulheres que desejam contracepção reversível de longo prazo. É indicado para lactantes, visto que não vai ocorrer interferência na qualidade e quantidade de leite e em mulheres que possuem contraindicação ao estrogênio, por exemplo. Além disso, não há contraindicação para a colocação do DIU de cobre após o aborto, podendo ser inserido em abortos de 1ํ trimestre, desde que não haja complicações prévias. Mulheres com ectopia cervical, neoplasia, cistos no ovário e HIV positivas também podem utilizá-lo (GIORDANO M, et al., 2015).

O DIU hormonal oferece alternativas para o tratamento de hemorragia, hiperplasia endometrial e adenomiose. Oferece melhoria dos sintomas e regulação do padrão menstrual de mulheres com endometriose e miomas uterinos (FEBRASGO, 2018). Segundo a Febrasgo (2018), a inserção do DIU de levonorgestrel é indicado para mulheres que sofrem com anemia devido à perda de grande volume de sangue devido ao ciclo menstrual, reduzindo esta perda em mais de $95 \%$ após um ano de uso. Ademais, pode ser usado para reposição hormonal nestas mulheres.

\section{Contraindicações}

Existem, relatadas pela Organização Mundial de Saúde (OMS), algumas contraindicações ao uso do DIU de cobre. Dentro da classificação de contraindicações absolutas, estão: gravidez, alergia ao cobre, câncer cervical ou endometrial, sépsis puerperal, Infecção Sexualmente Transmissível (IST) e doença inflamatória pélvica. Já nas contraindicações relativas, estão mulheres que possuem fator de risco para IST, mulheres imunocomprometidas e/ou que possuem câncer de ovário, doença trofoblástica benigna e aquelas com período pós-parto de 48 horas a 4 semanas (FEBRASGO, 2018).

Em relação ao DIU hormonal, são apontadas como contraindicações absolutas, gravidez confirmada ou suspeita, cervicite não tratada, doença hepática aguda ou tumor de fígado, alergia ao levonorgestrel e infecção aguda ou recente. Já como contraindicação relativa, tem-se: mulheres que não querem desenvolver oligomenorreia ou amenorreia, história de reflexo vasovagal, fator de risco importante para IST, história anterior de problemas com anticoncepção intrauterina, mulheres imunocomprometidas e sangramento uterino anormal não diagnosticado (FEBRASGO, 2018).

\section{Eficácia}

O DIU é um método com alta eficácia, com taxas de falhas parecidas com às da laqueadura (ADEYEMEFOWODE OA e BERCAW-PRATT JL, 2019). A taxa de eficácia do DIU hormonal é muito alta, podendo ser representada através do índice de Pearl 0,1. Portanto, possui excelente eficácia contraceptiva e apresenta desempenho equivalente quando usado de forma habitual e correta. A sua taxa de satisfação entre as mulheres mostrou índices superiores a $75 \%$ no primeiro ano de uso. Já o DIU de cobre, que é provavelmente o mais utilizado em todo mundo, tem durabilidade de dez anos e o índice de Pearl muito baixo, ocorrendo uma gravidez ou menos em cada 100 mulheres durante o primeiro ano de uso. Além disso, vários estudos mostraram que os dispositivos de cobre permanecem efetivos por 12 a 13 anos (FEBRASGO, 2016).

\section{Repercussões sistêmicas}

O DIU hormonal é um método altamente eficaz e possui inúmeros benefícios não contraceptivos, sendo o mais importante para tratamento de mulheres com sangramento menstrual intenso. Este efeito foi observado em estudos realizados, em que as usuárias apresentaram sangramento uterino mais leve, e além disso, cerca de $60 \%$ das mulheres viram uma melhora na hemoglobina e na dosagem de ferritina (BAHAMONDES L, et al., 2015). Já o DIU de cobre causa alteração na expressão gênica induzida pelo metal, que pode explicar um dos principais efeitos colaterais, como a hipermenorréia, devido a modificação da microvascularização subendometrial (DINEHART E, et al., 2020).

Nos estudos de Adeyemi-Fowode AO e Bercaw-Pratt JL (2019), analisaram 109 mulheres entre 18 a 30 anos que tiveram o DIU colocado em uma clínica de saúde estudantil, e foi observado que o DIU de cobre possui maior probabilidade de estar associado ao sangramento menstrual intenso e cólicas quando 
comparado ao DIU hormonal. Além disso, esta mesma pesquisa evidenciou que no fim do primeiro ano de uso do DIU hormonal, cerca de $20 \%$ das mulheres terão amenorreia, e dentro dos primeiros 2 anos mais de $50 \%$ das mulheres podem ter amenorréia ou oligomenorréia. Assim, o DIU hormonal pode ser mais aceitável nesta população devido ao seu padrão de sangramento menstrual mais favorável.

$\mathrm{Na}$ revisão sistemática proposta por Ti AJ, et al. (2020), relataram a interrupção do DIU devido ao sangramento ou dor. A descontinuação do DIU hormonal por sangramento ocorreu em cerca de 3,7 por 100 participantes e taxas de descontinuação por dor de 0,4 por 100 participantes, enquanto no DIU de cobre foram encontradas taxas de remoção para sangramento ou dor de 4,6 por 100 participantes. Dor e sangramento excessivo são as principais razões de descontinuação precoce do dispositivo (SANTOS PNS, et al., 2017).

Dor pélvica é outro efeito adverso relatado em usuárias de DIU. Em uma pesquisa com 132 mulheres que usavam anticoncepcionais reversíveis de ação prolongada, a dor pélvica foi associada a uma taxa maior de descontinuação do DIU (HOWARD B, et al., 2017). Da mesma forma, em um estudo de coorte retrospectivo com 307 usuárias adolescentes de DIU, a dor pélvica foi associada a uma taxa maior de descontinuação do DIU (DICKERSON L, et al., 2013).

A ocorrência de acne, aumento do peso, humor depressivo e cefaleia são alguns dos efeitos adversos causados pelo DIU hormonal, e que muitas das vezes não têm necessidade de ser retirado para tratarem tais repercussões. Os casos graves são bem raros, e a mulher deve ser orientada para fazer a remoção do dispositivo quando não houver uma melhora dos sintomas com o uso de espironolactona nos casos leves e moderados, ou uso de roacutan nos casos mais intensos (FEBRASGO, 2016)

No entanto, pesquisadores e médicos devem continuar na busca de práticas que minimizem os efeitos colaterais após a inserção do DIU, principalmente o de cobre, no que se diz respeito ao sangramento e a dor (SANDERS JN, et al., 2018). Um ensaio randomizado realizado por Hofmeyr GJ, et al. (2019), evidenciaram que o aumento na perda de sangue menstrual com o DIU de cobre foi reduzido com ibuprofeno, ácido tranexâmico e em menor grau, diclofenaco de sódio e o naproxeno foi considerado eficaz na redução da dismenorreia associada ao DIU.

\section{Complicações}

As taxas de expulsão dos dispositivos intrauterinos variam cerca de 2 a $10 \%$ para todas as usuárias. No estudo prospectivo com 1117 participantes, foram submetidas à inserção de DIU em uma clínica universitária e detectaram que houve uma taxa de expulsão de $3 \%$ em 6 meses após a colocação. Dados estes que corroboram que mulheres mais jovens têm menos probabilidade de expulsão quando comparadas às mulheres mais velhas (ADEYEMI-FOWODE OA e BERCAW-PRATT JL, 2019).

Um estudo canadense, proposto por Hsia JK e Creinin MD (2016), avaliou 51 mulheres por até 01 ano após a inserção do DIU de cobre. Cerca de $27 \%$ dessas mulheres tiveram o dispositivo expulso durante 0 primeiro ano, enquanto cerca de 16\% tiveram que remover o DIU devido a sintomas pélvicos. Das 21 mulheres que completaram 1 ano de uso, cerca de $29 \%$ estavam insatisfeitas com os efeitos colaterais.

Embora rara, a perfuração uterina é uma complicação séria associada ao uso do DIU. Foi descrito que isso ocorre a uma taxa de 0,3-2,5 a cada 1000 inserções do dispositivo hormonal e para o dispositivo de cobre, 0,3-2,2 a cada 1000. Neste mesmo estudo, o fator de risco mais significativo associado à perfuração foi a amamentação no momento da inserção, correspondendo 6,3 a cada 1000 participantes em mulheres que inseriram o DIU hormonal contra 3,7 a cada 1000 em mulheres que inseriram o DIU de cobre. E, além disso, a perfuração quando presente não trouxe lesões graves (BATUR P, et al., 2016).

Hofmeyr GJ, et al. (2010), também concluíram que o risco para usuárias do dispositivo de desenvolverem DIP é semelhante ao de mulheres que não usam contracepção. No entanto, apesar das preocupações existentes nas mulheres, os estudos evidenciam que o risco de DIP é baixo entre os usuários de DIU, porém, quando a infecção pélvica ocorre em mulheres com um DIU, o tratamento responde mais rapidamente se o dispositivo for removido (JATLAOUI TC, et al., 2017).

Além disso, o risco de perfuração uterina no momento da inserção do DIU em pacientes sem a presença de doença pélvica inflamatória corresponde entre 0 e $2 \%$, já quando a inserção ocorre com uma infecção não 
detectada, este número sobe de $2 \%$ para $5 \%$, para isso, mulheres sexualmente ativas devem ser rastreadas contra Neisseria gonorrhoeae e infecção por Chlamydia trachomatis no momento da inserção para reduzir esse risco (ADEYEMI-FOWODE OA e BERCAW-PRATT JL, 2019).

\section{Vantagens e desvantagens}

Para os critérios de elegibilidade de uso do DIU, a Organização Mundial de Saúde classifica os dispositivos como categoria 2 (vantagem supera o risco) para as nulíparas, enquanto as mulheres que já possuem filhos foram classificadas na categoria 1 (sem restrições). Dessa maneira, as recomendações evidenciam que a paridade não influencia o critério de elegibilidade dos dispositivos (MACHADO RB, 2017).

O dispositivo de cobre possui algumas vantagens em relação ao hormonal, sendo elas: ausência de hormônios, sem amenorreia, retorno da fertilidade imediato, redução de acidente vascular cerebral, entre outros (HOFMEYER GJ, et al., 2010). Além disso, possui eficácia como contracepção de emergência, tendo uma taxa de falha de menos de 1 por 1000 quando inserido dentro de 5 dias após a relação sexual, sendo mais significativo e mais eficaz do que qualquer contraceptivo hormonal (HSIA JK e CREININ MD, 2016).

Em relação às desvantagens do DIU de cobre, cita-se, principalmente, a inserção dolorosa e a requisição de um profissional qualificado para realizar o procedimento, aumento do fluxo menstrual e intensidade das cólicas, além de complicações raras, como a perfuração uterina e a migração do dispositivo (HOFMEYER GJ, et al., 2010).

O DIU hormonal apresenta benefícios além do método contraceptivo, como o tratamento do fluxo menstrual intenso, dismenorreia e é uma ferramenta que faz supressão da menstruação (MALMBORG A, et al., 2019). Além disso, é utilizado como off-label no tratamento da dor associada à endometriose em muitos países, e muitos estudos elucidam que esse tipo de tratamento é eficaz. Os sintomas mais comuns em pacientes com endometriose além da infertilidade, é a dismenorreia e dor pélvica (BAHAMONDES L, et al., 2015). A descontinuação do DIU hormonal está relacionada com algumas desvantagens que este método apresenta, como a amenorreia (considerada vantagem por algumas mulheres), atraso no retorno da fertilidade, ganho de peso, náusea, cefaleia, entre outros (HOFMEYER GJ, et al., 2010).

\section{Relação do DIU com Vírus da Imunodeficiência Humana (HIV)}

Marcus JL, et al. (2020), através de seus estudos, sugeriram um risco potencialmente aumentado para a aquisição de HIV com o DIU hormonal. Houve 89 diagnósticos de HIV no grupo DIU hormonal e 21 diagnósticos no grupo DIU de cobre. Porém, não houve nenhuma evidência que justificasse a maior probabilidade de contrair HIV usando o DIU hormonal quando comparado ao DIU de cobre.

Shanmugasundaram U, et al. (2016), notou alterações de caráter imunológicas e inflamatórias locais com o uso do DIU hormonal, como o aumento da quantidade de células T no colo do útero e no endométrio, levantando preocupações sobre o potencial aumento do risco de HIV. Em contrapartida, Achilles SJ, et al. (2014), observaram uma diminuição das células T que expressam receptores de quimiocina CC, correceptor do HIV no colo do útero em pacientes com DIU hormonal, sugerindo que o risco de HIV não é elevado.

\section{Interferência na fertilidade após a remoção do dispositivo}

O retorno da fertilidade logo após a remoção do DIU tem sido bem discutido na literatura, no entanto, existem algumas controvérsias sobre quando a fertilidade retorna e se as modalidades de DIU afetam isso de maneira diferente. Vários estudos mostram que as mulheres são capazes de engravidar após a remoção do DIU, seja ele de cobre ou hormonal, dentro de um período de 12 meses após a remoção (DINEHART E, et al., 2020).

Um ensaio clínico randomizado realizado por Hov GG, et al. (2007) analisaram 205 mulheres que utilizaram o DIU, e observaram que não houve evidências que comprovem que o uso prévio de DIU de cobre aumente o risco de infertilidade, seja por qual foi o motivo da remoção, se por decisão para programar a gestação ou por complicações. Neste mesmo estudo concluíram que o tempo necessário para a concepção não foi afetado pelo tempo de uso do dispositivo. Já Dinehart E, et al. (2020), analisaram 179 mulheres que decidiram 
interromper o uso do DIU hormonal para gestarem e cerca de $37,4 \%$ conceberam nos primeiros 3 meses e $71,2 \%$ conceberam em 12 meses. Em contrapartida, um estudo de coorte proposto por Doll $\mathrm{H}$, et al. (2001) evidenciaram que o uso prolongado de DIU parece estar relacionado com um comprometimento da fertilidade, fazendo relação diretamente com o tempo de uso.

\section{CONSIDERAÇÕES FINAIS}

Mediante a análise bibliográfica deste estudo, conclui-se que os dispositivos intrauterinos hormonais e de cobre apresentam características específicas em relação à sua indicação, eficácia, repercussões sistêmicas e principalmente os efeitos colaterais. Dismenorreia, hipermenorreia, perfuração uterina, expulsão, surgimento de doenças inflamatórias pélvicas demonstrou estar intimamente relacionado às principais complicações do método. Por outro lado, quando é bem indicado e inserido por um profissional qualificado, torna-se um dos métodos com o menor índice de falha no que se refere à gravidez indesejada. Dessa forma, observa-se que ambos DIU's apresentam prós e contras, cabendo ao médico priorizar para aquela mulher qual dispositivo o benefício supera os malefícios no que tange ao desejo da paciente, podendo ser a contracepção, regulação do ciclo menstrual ou até mesmo o tratamento de patologias.

\section{REFERÊNCIAS}

1. ACHILLES SL, et al. Impact of contraceptive initiation on vaginal microbiota. American Journal of Obstetrics \& Gynecology, 2018; 218(6): 1-10.

2. ACHILLES SL, et al. Mudanças nas populações de células imunes do trato genital após o início da contracepção intrauterina. Am J Obstet Gynecol, 2014;211: 489 e1-9.

3. ADEYEMI-FOWODE OA, BERCAW PRATT JL. Intrauterine Devices: Effective Contraception with Noncontraceptive Benefits for Adolescents. J Pediatr Adolesc Gynecol, 2019; 32: 2-6.

4. ARROWSMITH ME, et al. Interventions for increasing uptake of copper intrauterine devices: systematic review and meta-analysis. Contraception, 2012; 86: 600-605.

5. BAHAMONDES L, et al. Non-contraceptive benefits of hormonal and intrauterine reversible contraceptive methods. Human Reproduction Update, 2015; 0(0): 1-12.

6. BATUR P, et al. Contraception: Efficacy, Risks, Continuation Rates, and Use in High-Risk Women. Journal of Women's Health, 2016; 25(8): 1-4.

7. DICKERSON L, et al. Satisfaction, early removal, and side effects associated with long-acting reversible contraception. Fam Med, 2013; 45:701

8. DINEHART E, et al. Levonorgestrel IUD: is there a long-lasting effect on return to fertility? Journal of Assisted Reproduction and Genetics, 2020; 37: 45-52.

9. DOLL H, et al. Return of fertility in nulliparous women after discontinuation of the intrauterine device: comparison with women discontinuing other methods of contraception. BJOG. 2001; 108: 304-14.

10. FEBRASGO. Contracepção reversível de longa ação. -- São Paulo: Federação Brasileira das Associações de Ginecologia e Obstetrícia (FEBRASGO), 2016.

11. FEBRASGO. Manual de anticoncepção. -- São Paulo: Federação Brasileira das Associações de Ginecologia e Obstetrícia (FEBRASGO), 2018.

12. GIORDANO M, et al. Dispositivo intrauterino de cobre. FEMINA, 2015; 43(Suppl. 1).

13. MARCUS JL, et al. Use of Intrauterine Devices and Risk of Human Immunodeficiency Virus Acquisition Among Insured Women in the United States. Clinical Infectious Diseases, 2020; 70(10): 2221-2223.

14. HOFMEYR GJ, et al. Copper containing intra-uterine devices versus depot progestogens for contraception. Cochrane Database of Systematic Reviews, 2010; 6: 1-23.

15. HOV GG, et al. Use of IUD and subsequent fertility - follow-up after participation in a randomized clinical trial. Contraception, 2007; 75(88-92): 23.

16. HOWARD B, et al. Trends in use of and complications from intrauterine contraceptive devices and tubal ligation or occlusion. Reprod Health, 2017; 14:70

17. HSIA JK, CREININ MD. Intrauterine Contraception. Semin Reprod Med, 2016; 34: 175-182.

18. JATLAOUI TC, et al. The safety of intrauterine devices among young women: a systematic review. Contraception, 2017; 95(1): 17-39.

19. MACHADO RB. Uso de dispositivos intrauterinos (DIU) em nulíparas - São Paulo: Federação Brasileira das Associações de Ginecologia e Obstetrícia (FEBRASGO), 2017.

20. MALMBORG A, et al. A survey of young women's perceptions of the influence of the Levonorgestrel-Intrauterine System or copper-intrauterine device on sexual desire. Sexual \& Reproductive Healthcare, 2019; 21: 75-80.

21. SANDERS JN, et al. Two-year continuation of intrauterine devices and contraceptive implants in a mixed-payer setting: a retrospective review. Am J Obstet Gynecol, 2017; 216(6): 1-8.

22. SANTOS PNS, et al. Changes in Body Composition in Women using Long-acting Reversible Contraception. Contraception, 2017; 95(4): 382-389.

23. SHANMUGASUNDARAM U, et al. Efeitos do dispositivo intrauterino de liberação de levonorgestrel no microambiente imunológico do colo uterino e endométrio humano. Am J Reprod Immunol, 2016; 76: 137-48.)

24. STANFORD JB, MIKOLAJCZYK RT. Mechanisms of action of intrauterine devices: Update and estimation of postfertilization effects. Am J Obstet Gynecol, 2002; 187: 1699-1708.

25. TI AJ, et al. Effectiveness and safety of extending intrauterine device duration: a systematic review. American Journal of Obstetrics \& Gynecology, 2020; 223(1): 24-35. 